\title{
Classifying Compliant Manipulation Tasks for Automated Planning in Robotics
}

\author{
Daniel Leidner ${ }^{1}$, Christoph Borst ${ }^{1}$, Alexander Dietrich ${ }^{1}$, Michael Beetz ${ }^{2}$, and Alin Albu-Schäffer ${ }^{1,3}$
}

\begin{abstract}
Many household chores and industrial manufacturing tasks require a certain compliant behavior to make deliberate physical contact with the environment. This compliant behavior can be implemented by modern robotic manipulators. However, in order to plan the task execution, a robot requires generic process models of these tasks which can be adapted to different domains and varying environmental conditions. In this work we propose a classification of compliant manipulation tasks meeting these requirements, to derive related actions for automated planning. We also present a classification for the sub-category of wiping tasks, which are most common and of great importance in service robotics. We categorize actions from an object-centric perspective to make them independent of any specific robot kinematics. The aim of the proposed taxonomy is to guide robotic programmers to develop generic actions for any kind of robotic systems in arbitrary domains.
\end{abstract}

\section{INTRODUCTION}

Probably the most essential aspect of any manipulation task is the effect to the environment. Artificial intelligence (AI) research has shown how this can be properly taken into account in automated planning systems, where actions are described based on their pre-conditions and effects [1]. Unfortunately, this aspect has been mostly abandoned in robotics research. Traditionally, robots are clueless about the purpose of their motions, and they are not aware of the resulting changes to the world. A similar trend can be observed in research on the classification of manipulation tasks. Traditional taxonomies found in the literature usually apply hand-centric views and classify by finger position [2], relative motions [3], or geometric dimensions [4]. The applications and effects are mostly neglected. We argue that a novel point of view has to be applied to classify actions on a high level of abstraction according to their effects to the physical world. Based on this, less abstract sub-categories can be defined to derive generic process models and to combine symbolic and geometric parameters.

We are especially interested in physically compliant manipulation tasks, where a dedicated workspace trajectory is executed with a certain stiffness, while a limited amount of force is exerted. For example, a robot might be commanded to solve the task of sweeping shards of a broken mug by the use of a broom as illustrated in Fig. 1. This is a typical household chore which requires not just detailed geometric parameterization, i.e. how to handle the broom

1 Institute of Robotics and Mechatronics, German Aerospace Center (DLR), Wessling, Germany. Contact: daniel.leidnerdalr.de

2 Institute for Artificial Intelligence, University of Bremen, Bremen, Germany.

3 Sensor-Based Robotic Sys. and Intelligent Assistance Sys., Technische Universität München (TUM), Garching, Germany.

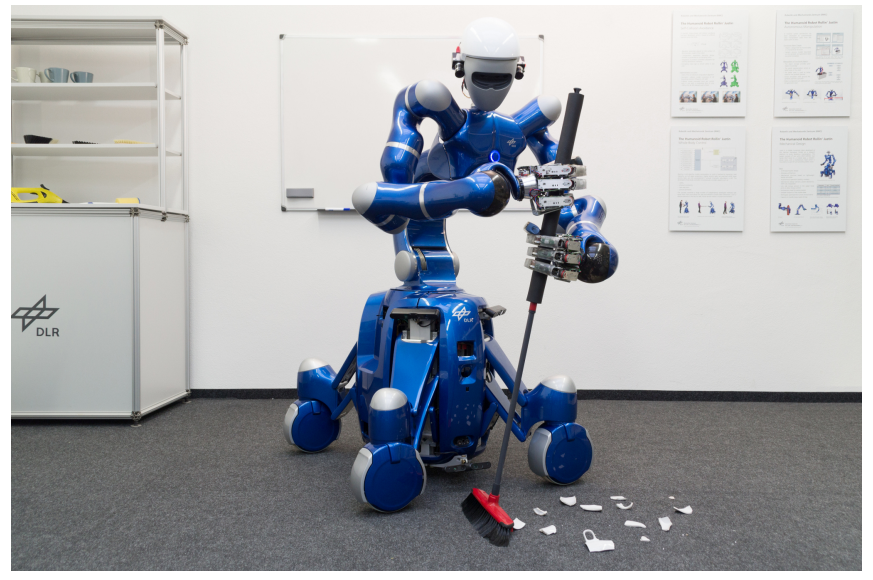

Fig. 1. The humanoid Rollin' Justin sweeping shards of a broken mug as example for a compliant manipulation task.

and how to contact the floor, respectively the shards, but also symbolic parameterization. That is, what are the preconditions and effects of the action, what is the goal and what role have the shards. To classify actions accordingly, a taxonomy considering both, symbolic and geometric aspects is demanded. The deployed robot should be seen as a tool to solve the manipulation task. It has no influence on the task parameters and is therefore secondary for a classification.

In this work we propose a classification of compliant manipulation tasks based on symbolic effects to the environment. The classification terms are defined w.r.t. the contact situation between the tools/objects and the target/environment. Additionally, we conduct a subcategorization on wiping tasks, which constitute the main class of common household chores. By exploiting similarities in geometric structures of wiping tasks we are able to derive generic process models, independent of any specific kinematics and therefore applicable to arbitrary robots. Finally we demonstrate how to concretize actions from this two-step classification based on the example at hand, i.e. sweeping shards with a broom. Our long term goal is to develop a goaloriented set of generic manipulation actions to finally bridge the gap between AI planning and robotic manipulation.

The paper is organized as follows: After a review on the literature, we introduce our classification of compliant manipulation tasks in Sec. III. A classification on the subcategory of wiping tasks will be detailed in Sec. IV. Based on our findings we demonstrate the application of our taxonomy to derive and parameterize suitable actions for automated planning in Sec. V. We conclude with a discussion on the classification in Sec. VI. 


\section{RELATED WORK}

Several taxonomies for classifying object manipulation have been developed in the past. They can be separated into grasp taxonomies and manipulation taxonomies.

Grasp taxonomies have been widely adopted in robotics for grasp planning [5] and the design of anthropomorphic robotic hands [6]. The taxonomy of Kapandji [7] was originally developed to evaluate grasping capabilities of human hands but can also be used to determine the performance of anthropomorphic robotic hands [8]. The taxonomy of Cutkosky [2] is probably the most well-known in robotics. It was designed after studying several machining tasks and consists of 16 grasp types ordered in a hierarchical tree. It mainly distinguishes between power grasps and precision grasps. Based on these and many other taxonomies, a comprehensive grasp taxonomy was developed by Feix et al. [9]. It captures over 33 classified grasps. We argue that pure grasp taxonomies are hard to apply to automated planning since a performed action cannot directly and exclusively be related to a certain grasp type. Furthermore, the classified grasps are considered to be executed by a human or an anthropomorphic robotic hand, and are therefore not suited for industrial robotic manipulators possibly only providing a two-jaw gripper, or no gripper at all but exchangeable tools.

Manipulation taxonomies differ from grasp taxonomies by not purely defining classes based on the configuration of a hand, but also considering the objects to be manipulated. The hand-centric taxonomy of Bullock et al. [3] classifies human manipulation according to the relative motion of the hand w.r.t. the grasped object during task execution. Similar to the classification of Cutkosky, Bullock et al. define a hierarchical tree structure to distinguish different classes of prehensile and non-prehensile manipulation. Additionally they provide a taxonomy on in-hand manipulation related to translations and rotations of the object in hand. The taxonomy of Bloomfield et al. [10] classifies haptic actions by the applied forces and torques w.r.t. the orientation of human hands. These parameters depend on the object position in hand and are therefore hardly applicable to arbitrary robotic manipulators. Worgotter et al. [11] consider the relation between objects in space and time to classify manipulation actions in assembly and dis-assembly tasks. This allows for a symbolic classification but the domain is limited. An objectcentric classification has been conducted by Morrow et al. [4]. They developed a set of low-level primitive actions based on geometric definitions to compose more complex actions. Although the primitives can be used for scheduling robot motions, this level of abstraction is not suitable for symbolic planning. A classification of contact situations was conducted by Vukobratović and Veljko [12] to model the dynamics of contact tasks. Among others, the authors model reaction force, impact, contact friction, and deformation. However, instead of classifying tasks and parameterize them accordingly, they suggest to unify all models to cover all the effects mentioned. It is arguable if this can result in performant task execution for every case.
Liu et al. [13] try to combine traditional grasp taxonomies with manipulation taxonomies by observing grasps in action. They do not only classify finger joint positions but also annotate each grasp with common English words to describe the action type. Furthermore, they argue that the parameters required to define a grasp in action are similar to the parameters required to define impedance behavior or motion/force/stiffness properties of robotic manipulators. We agree with them in this point, but believe that the symbolic representation of an action is essential and should be considered in a classification as well.

\section{Classification OF Compliant Manipulation}

Recent advancements in mechanical robot design and control enable the systems to get in soft contact with their environment. However, the deployed robots can vary significantly. Therefore, it is mandatory to develop reliable, flexible, and generic robot actions, independent of a robot specific kinematics (e.g. human hand kinematics). One way to overcome this issue is to develop robotic manipulation actions from an object point of view rather than relying on the robot capabilities. With this in mind we have developed a hybrid reasoning system combing AI-based planning mechanisms [14] and compliant robotic manipulation [15]. We have successfully tested this approach in several household chores, ranging from simple fetch-and-carry tasks to compliant whole-body manipulation such as cleaning large windows [16]. However, to minimize the development effort and to create sustainable software modules, actions have to be arranged in a higher level of abstraction. Therefore, we propose to classify compliant manipulation tasks w.r.t. the effects to the world on a symbolic level of abstraction and not solely based on geometric features and low-level control properties.

Our manipulation taxonomy is illustrated in Fig. 2. Similar to the classifications of Cutkosky [2] and Bullock et al. [3] we apply a hierarchical tree structure to guide the reasoning process. Depending on the depth in the tree, a robot has to reason in more detail about the required parameters (see Sec. III-C). Instead of developing our taxonomy based on kinematics features, we propose a symbolic view w.r.t. the objects involved in the tasks. Therefore, our taxonomy characterizes actions in a descriptive manner to make it complementary to the action definition in automated planning [1]: Each branch defines a symbolic classifier related to the contact situation between an object or tool and a target in the environment, without limiting how the contact is exerted in particular. In the follow-up, generic process models can be derived by sub-categorizing each leaf node as done in Sec. IV. Consequently, a concrete implementation based on the classification has to integrate the respective parameters accordingly, which is outlined in Sec. V. Note that we illustrate the actions executed with a human hand although our classification is not limited to a specific manipulator or hand. Similarly we do not distinguish between one or more manipulators. The classification terms and the example actions are detailed in the following sections. 
COMPLIANT MANIPULATION TASKS

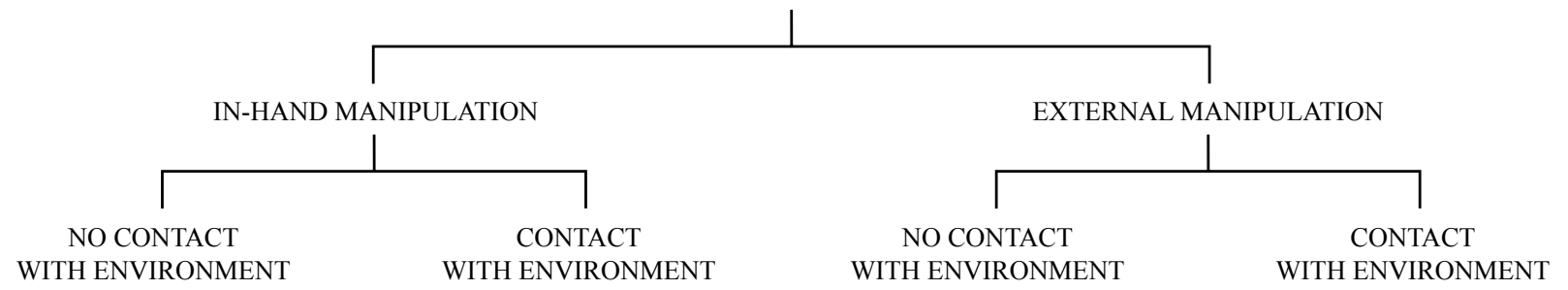

WITH ENVIRONMENT

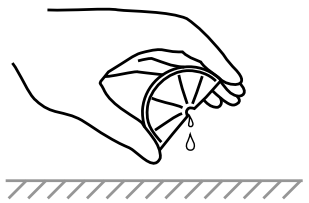

Squeezing a lemon
WITH ENVIRONMENT

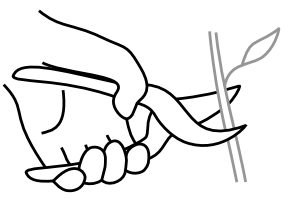

Cutting with a shear
WITH ENVIRONMENT

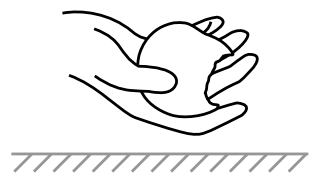

Holding an object
WITH ENVIRONMENT

TASK-IRRELEVANT

CONTACT FRICTION

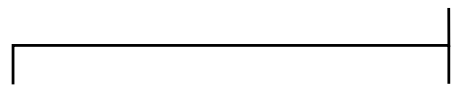

NEGLIGIBLE

DEFORMATION

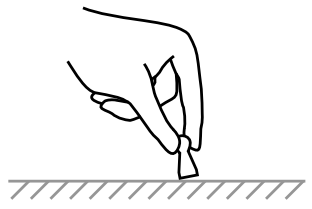

Placing an object

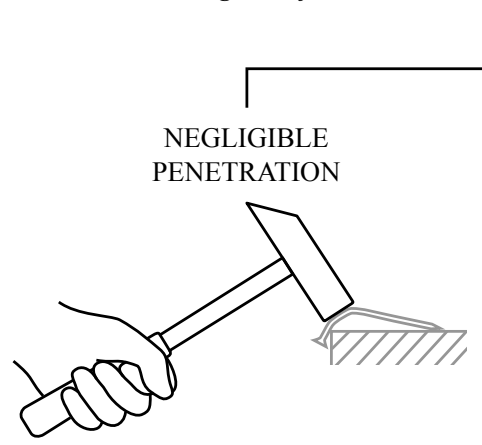

Forging with a hammer
SUBSTANTIAL

DEFORMATION

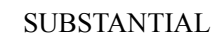

PENETRATION

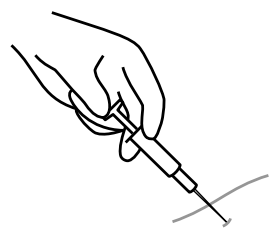

Injecting a syringe

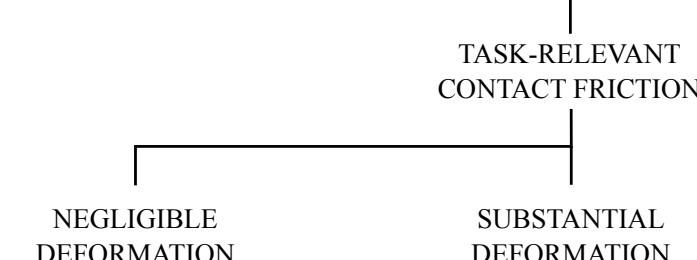

DEFORMATION

DEFORMATION

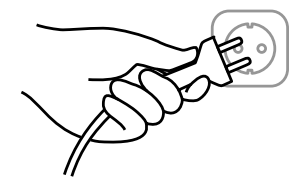

Connecting a plug

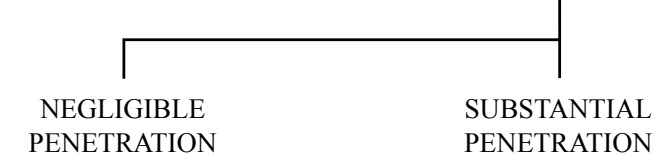

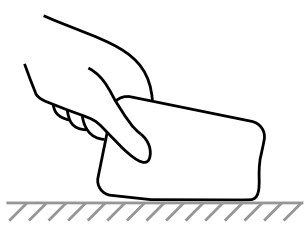

Cleaning with a sponge

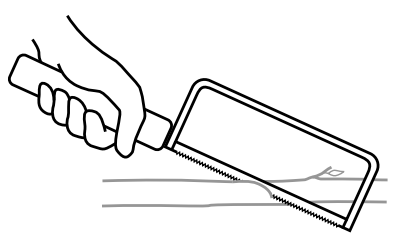

Cutting a branch with a saw

Fig. 2. Tree hierarchy of compliant manipulation tasks. For each leaf of the tree an example action is given. The two reference systems describing the contact situation are the hand-object system, colored in black, and the environment, colored in gray. The complexity increases from top to bottom in number of parameters to be considered during the reasoning process. The illustrated hand is representative for arbitrary robotic manipulators. Please note that we do not consider hand kinematics, the hand posture, nor the contact between the hand and the grasped object in our classification.

\section{A. Classification Terms}

This section describes the terms of our taxonomy. The classifiers are selected to categorize abstract action classes for automated planning. With each classifier, additional information is available to reason about the underlying effects to the environment, an adequate set of parameters, and the required control strategies (see Sec. III-C).

Since our approach is object-centric, each classifier is chosen w.r.t. the nature of the contact between the two reference systems, namely the hand-object system (black) and the environment, respectively the target object (gray).
In-Hand/External: The first classifier defines whether the force is exerted within the manipulator or hand (inhand) or whether it originates from an external source in the environment (external). This term is related to the concept of virtual linkage introduced by Williams and Khatib [17], which defines internal forces as forces within the grasp map of a manipulator.

Contact: This term defines whether the hand-object system makes deliberate contact with the environment (resulting in reaction force) and whether a proper set of compliant control parameters is crucial for the task performance. Force 
also originates without contact by acceleration (e.g. by gravity) of a mass, which also includes simply holding an object, for example.

Contact Friction: Contact friction is observed when an object is moved along the surface of another object in contact. Force has to be exerted along the surface in order to overcome the friction. Friction may be irrelevant for certain manipulation tasks with small surfaces and is negligible then. It can also be a substantial part of an action with relevance for the task, as for example in cleaning tasks. Tasks involving friction are very sensitive to the direction of motion which may require different stiffness settings for individual Cartesian directions [18], [16]. As opposed to this, force is mainly exerted in other ways e.g. pressure, torque or upon impact, if friction is negligible.

Deformation: Non-rigid object manipulation requires to reason about the deformation of objects during the contact phase, but also afterwards if the deformation is persistent. Deformations occur either in the object to be manipulated, or the environment, respective, the target object. Geometric deformation may result in a different symbolic state for an object, e. g. it can be an indicator for damage. Note that every contact results in deformation in microscopic scale which is neglected for our classification.

Penetration: Penetration occurs if the target structure cannot resist the applied force anymore. Penetration may lead to significant, irreversible alteration of the geometric state of an object including the full separation into multiple parts. This has to be considered on the symbolic level. Research on tool-soil interaction has proven that active variable force and stiffness is necessary while deliberately penetrating an object (soil) due to the varying resistance force [19]. Note that any deformation can involve penetration in microscopic scale which is neglected for our classification.

\section{B. Example Actions}

The provided example actions are representative for the combinations of the available parameters and discussed in the following. As the taxonomy is object-centric, the classification terms describe effects as relations between the handobject system (black in Fig. 2) and the environment (gray in Fig. 2). Please note that the classification terms are shortened in the headings below for readability.

In Hand - No Contact: There is no contact between the hand-object system and the environment. Forces only occur intrinsic to the hand (in-hand), e.g. squeezing a lemon or moving a pen in-hand.

In Hand - Contact: The hand-object system is in contact with the environment. In-hand manipulation results in a force intrinsic to the manipulator, e.g. cutting with a shear or using a screwdriver.

External - No Contact: The force originates from accelerating a mass/inertia where the acceleration also involves gravity, e.g. holding or lifting an object.

External - Contact - Irr. Friction - Neg. Deformation: Classical rigid contact with rigid body motions where force is transfered as e.g. pressure, torque, or impact, e.g. placing or touching an object.

External - Contact - Irr. Friction - Deformation Neg. Penetration: Deformation occurs upon contact where friction is of lesser relevance to the task, i.e. pushing, pulling, bending, or hitting something, e.g. forging iron with a hammer or folding paper.

External - Contact - Irr. Friction - Deformation Penetration: A rigid object deliberately penetrates a nonrigid object with irreversible effect. Friction is significantly smaller than the resistance force, e.g. injecting a syringe or fork up food.

External - Contact - Friction - Neg. Deformation: A rigid object is in physical contact with another rigid object, guided by the contours of the objects in a sliding motion, e. g. connecting a plug or inserting a key.

External - Contact - Friction - Deformation - Neg. Penetration: Guiding a soft object along the surface of a rigid object or vice versa. Also both objects can be soft. Usually the task involves a medium, which can be considered to be non-rigid in a macroscopic view. Most of these actions can be summarized as wiping tasks, to be detailed in Sec. IV, e. g. cleaning a window with a window wiper, ironing a shirt, or painting a wall.

External - Contact - Friction - Deformation - Penetration: Upon sliding contact, a rigid object penetrates the target deliberately. The penetration effect is irreversible, e. g. sawing a branch or cutting bread.

\section{Discussion}

Each classification term provides additional information for the reasoning process and has to be considered during the task execution. According to the literature [17], [19], [20], [21], [22], [23], especially the required control strategies have to be selected w.r.t. to the nature of the contact: For example, squeezing a lemon mainly relies on compliant inhand control strategies such as [17], [20], whereas inserting a plug can be efficiently solved with Cartesian compliance at the end-effector plus an appropriate strategy to prevent the plug from jamming [21]. A task without penetration can exploit impedance-control with a dedicated stiffness to get in soft contact and wipe along a surface [22], which can be applied to window wiping, for example [16]. If penetration is a substantial part of the action, force has to be adapted according to the penetration depth [19] or as soon as the penetration occurs [23]. The varying requirements have to be taken into account by a properly parameterizable control framework [15], [16]. The control parameters depend thereby on the physical parameters (e.g. mass, inertia, or center of mass) of the objects involved in the task execution.

A generic classification can sometimes result in ambiguous cases where an action cannot be assigned to one particular class. For example, some tasks may or may not involve substantial friction depending on the actual state of the environment e.g. pushing a door. In some cases inaccurate parameterization can lead to undesired effects. For example, if a sponge is used to clean a knife, too much force might 
lead to a entirely different action, namely cutting the sponge with the knife. In fact it is notable that the objects involved in the task execution and the current environmental conditions always influence the task parameters, independent of any classification and should therefore always be considered during the reasoning process. Furthermore, many tasks can only be described by combining multiple branches of the tree. Especially handling electric tools requires to activate a button in-hand while exerting force with the tool to the environment, such as drilling a hole with an electric drill.

In general, the proposed classification can describe compliant manipulation tasks on a high level of abstraction. However, it is too abstract to directly implement concrete actions from it, since the classification terms only define the nature of the contact symbolically, and not how the contact is geometrically established. Therefore, we propose a twostep approach to classify compliant manipulation tasks on both, symbolic and geometric levels of abstractions likewise. Accordingly, each leaf illustrated in Fig. 2 has to be revisited to extract similarities to derive generic process models. We have conducted this sub-categorization for the category of wiping tasks in the following section.

\section{Classifying Wiping Tasks and Deriving PROCESS MODELS}

Our main classification described in the previous section does not consider geometric parameters, but rather more abstract symbolic parameters. However, to create a connection between automated symbolic planning and geometric planning and parameterization of robotic manipulation tasks, the abstract definitions have to be concretized. According to the analysis of Cakmak et al. [24], we conduct this subclassification exemplary for the category of the most frequent household chores. The authors have analyzed daily chore lists and found out that cleaning tasks are the most frequent household chores. In particular, $49.8 \%$ of the investigated tasks were related to wiping surfaces of objects, furniture, or rooms. Similar to us, Cakmak et al. argue that "tasks within a certain category exhibit similar structures that can be exploited while implementing robotic capabilities" [24], where they explicitly address cleaning tasks.

In addition, we observed a wide variety of cleaningunrelated tasks based on the principle of wiping a surface, e.g. painting a wall. Besides the tool-surface contact, wiping tasks share another common component, namely the medium (particles/liquid between tool/surface, e.g. dust or paint). In summary, these tasks can be formulated as guiding $a$ tool along a target surface while maintaining contact to manipulate some sort of medium. Wiping fits in the group of External - Contact - Friction - Deformation - Neg. Penetration w. r. t. our classification (see Fig. 2). Accordingly, mainly friction and deformation have to be taken into account by the control strategy in contact. The actions, however, differ in their geometric process models. So does sweeping with a broom require directed motions, while the trajectories in vacuuming maybe random in general. The actions have to be distinguished carefully in order to deal with these differences in a generalized way. We conduct a sub-categorization to extract geometric process models for the different types of wiping. De facto we conduct a two-step classification to incorporate symbolic and geometric properties similarly.

Similar to the contact analysis in the main classification, we do not directly categorize wiping tasks w. r. t. geometric features, but rather classify them based on symbolic effects to implicitly group actions with similar geometric structures. We investigate the tool - surface - medium 3-tuples, where the final geometric state of the medium corresponds to the desired symbolic goal state. Based on this role of the medium, individual process models can be derived. We are able to identify nine action types related to specific wiping tasks. The actions are grouped in the procedures of applying, removing, and modifying the medium. Additionally, a toolcentric view, a surface-centric view, and a medium-centric view are applied to categorize the actions. The resulting matrix structure is illustrated in Fig. 3 and explained in detail from the top left to the bottom right:

Absorb: A medium is absorbed upon close vicinity to the tool. This may be caused due to electrostatic force as known from dusting, an air draft from a vacuum, or capillary action as seen between a sponge and water. The effect is mostly unrelated to the direction of motion.

Skim: The final location of the medium is not of interest and is therefore illustrated as skimmed from the surface. Scratching ice from a car window is considered as skimming. The direction of motion is defined by the individual geometric topology.

Collect: Collecting can be related to skimming. However, the medium has to be collected afterwards, e.g. to remove it accumulated. Exemplary actions are collecting leaves with a rake or sweeping up shards. The tool alignment w.r.t. the surface is crucial.

Emit: Emitting is the counterpart to absorbing. The medium is initially located on or in the tool and is applied to the surface as it is done for painting a wall, for example. Typically, the whole surface is involved.

Distribute: Distributing a medium is related to emitting a medium. However, the medium is already located on the surface. Applying shoe polish is such a task. The task trajectory is important to distribute the medium on the surface.

Process: Processing is a medium-centric action. The medium is used to alter the surface on purpose, as done with cement, for example. It is also possible that the surface is only used to directly manipulate the medium as done with cookie dough.

Scrub: Scrubbing merges an auxiliary medium with an unwanted medium (e.g. detergent and dirt) by exerting force under repetitive motions to remove the unwanted medium. Many cleaning tasks can be categorized as scrubbing, e.g. scrubbing the oven, scrubbing a pan, or scrubbing the floor.

Grind: Grinding is often used in manufacturing such as planing wood. The medium is separated from the surface and is often a waste product. The tool alignment is crucial for the result. Note that grinding of a surface is considered as negligible microscopic penetration here. 


\section{CLASSIFICATION OF WIPING TASKS}

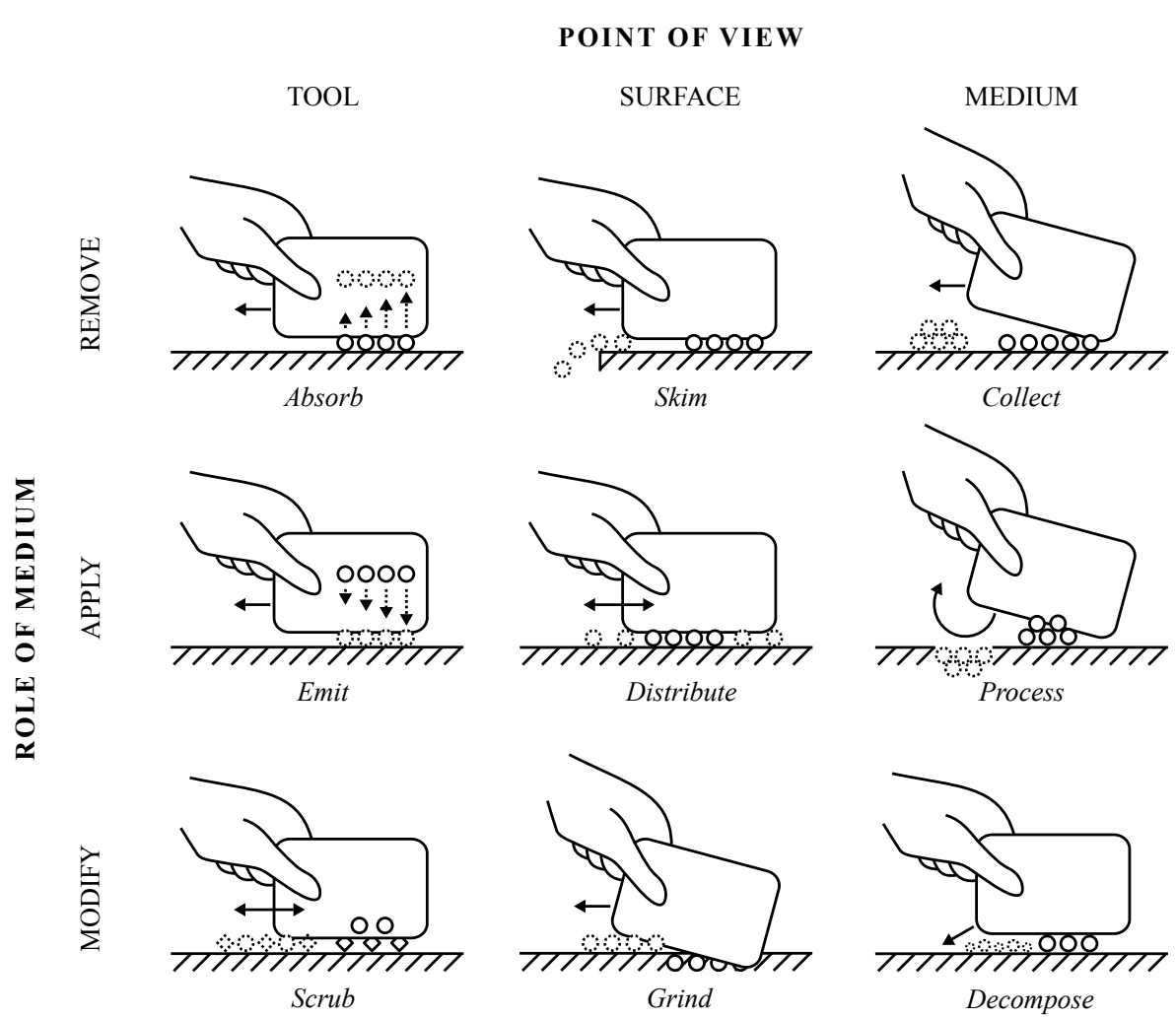

Fig. 3. Matrix classification of wiping tasks according to the tool, the surface, and the medium, versus the role of the medium. The tool (exemplarily grasped by a human hand) is abstracted as a rectangle capable of all illustrated actions. The motion of the tool is indicated as a solid arrow. The surface is always shown on the bottom of the corresponding matrix cell. It might be flat as illustrated, curved, or of any other shape. The medium is shown in the initial state (solid circles) and in the goal state after the action is performed (dashed circles) where dashed arrows indicate the transition of the medium.

Decompose: Decomposing splits the medium into smaller particles. More iterations can possibly lead to smaller particles. Pestle with a mortar is one example.

Fig. 3 illustrates the versatility of wiping actions. For each action, numerous tools might be suitable to achieve the desired goal. As for the main classification it is possible that not all tasks can be described by only one process model. It is also possible that one particular chore is actually a combination of several wiping actions such as mopping, which is a sequential combination of emitting, distributing, scrubbing, and absorbing.

The topology of a task can influence the parameter ranges of the executed action. For example, the force required to move a medium is related to the medium size paired with the surface friction, e.g. smaller shards are harder to remove from a carpet than bigger ones. Also, time has a significant effect on some tasks. For example, if a mug is cleaned right after it was used, coffee leftovers can be removed with little effort. If a mug is cleaned the next day, the required force will be higher and more iterations are necessary to remove the leftovers. Hard-coding parameters is inappropriate. Instead, a process model has to incorporate a proper parameterization. The task parameters can be efficiently stored in an objectcentric prior knowledge-base. In the particular example of wiping tasks, the Cartesian motion, the Cartesian stiffness and the maximal Cartesian force have to be stored w.r.t. to the tool - surface - medium combination.
In the following section we demonstrate how the proposed classifications can be utilized to derive concrete actions in order to solve the related tasks w.r.t. the individual environmental conditions.

\section{IMPLEMENTING MANiPUlation ACTIONS FOR Automated Planning}

We showcase the application of our taxonomy at the example of sweeping shards of a broken mug as illustrated in Fig. 1. As for any compliant manipulation task, but especially for cleaning tasks, an adequate parameterization is mandatory for a performant task execution. To achieve this, a knowledge-based approach has been developed [14] to parameterize actions w.r.t. the objects involved in the task and the current world state. The framework is based on hybrid reasoning, which combines symbolic and geometric reasoning to plan the task execution. The task knowledge is thereby stored in so-called action templates, defining both the symbolic action description, as required for automated planning, and also the geometric process model. Action templates define a concept which is very well suited to define versatile process models. We utilize them to demonstrate the application of our taxonomy. Please note that we only outline the conceptual application in the scope of this work here. For a detailed description of action templates please refer to [14].

The sub-categorization of wiping tasks as defined in Fig. 3 allows us to define one action template for each 
task. We derive a symbolic effect verb catalog including $a b$ sorbed, skimmed, collected, emitted, distributed, processed, scrubbed, ground, and decomposed. These symbolic descriptors are applicable as predicates as defined in the Planning Domain Definition Language (PDDL) [25], the standard language for automated planning. They are utilized to describe preconditions and effects for symbolic planning. PDDL is used to define the desired state of the medium in the symbolic header of an action template. Based on this representation, a symbolic planner can schedule the required actions to solve the tasks w.r.t. the target surface and the tools provided.

PDDL is commonly used in automated planning to model physical states in an abstract form. However, it is limited to a descriptive vocabulary and unable to fully describe physical state transitions in detail. A purely symbolic description of effects is therefore to abstract to solve for a desired physical goal state. The state collected, for example, can be solved in different ways depending on the objects involved in the task execution and their geometric topology. The robot has to reason about the desired physical situation and infer the steps to achieve a satisfactory goal on its own. Furthermore, some states may actually describe completely new geometric situations where new objects are created or destroyed (e.g. decomposed). To overcome this issues, the symbolic representation has to be grounded by utilizing commonsense reasoning methods. One way is to apply qualitative and quantitative reasoning as discussed in the Qualitative Process Theory [26], which was recently utilized to parameterize robotic manipulation actions [27]. Another approach is to simulate the physical behavior based on Naive Physics [28], which has been applied to the problem of absorbing water with a sponge in a robotic manipulation scenario [29], for example. The appropriate approach is thereby depending on the individual task.

The symbolic goal of sweeping shards is to collect the shards in one spot. A matching geometric process model needs to be defined based on the geometric topology of the task. Each process model has to be parameter-independent, meaning it should be generic to solve a wide variety of tasks. In the example at hand, the collect action has to be applicable to collecting shards with a broom, collecting leaves with a rake, or collecting snow with a plow likewise. The distribution of the medium (shards, leaves, or snow) defines the region of interest which is used together with the curvature of the surface to compute a set of workspace trajectories for the respective tool center points (TCP). Each trajectory is directed to collect the medium in one particular spot. These workspace trajectories are interpreted at run-time by the modules provided by the deployed robot (e.g. inverse kinematics, motion planning, and navigation modules). Additionally, the robot control framework is parameterized w. r. t. the properties of the involved objects. For example, the applied stiffness and the maximum force can be related to the medium size, the compliance (deformation) of the tool, and the resulting friction. Object properties can be mined from the web [30], shared between robots [31], or manually defined by the programmer, for example.
The task of collecting shards of a broken mug was performed by the humanoid robot Rollin' Justin in the video [32]. Similarly, skimming a window and the task of scrubbing a mug was performed. Each of the actions requires an individual parameterization w.r.t. the properties of the objects involved in the task execution. Among others, the Cartesian task trajectory, the Cartesian task stiffness, and the allowed maximum Cartesian forces have to be defined. A complementary analysis on this connection between highlevel task reasoning and low-level robot control has been conducted in our previous work [33]. The tasks were implemented as action templates based on a preliminary version of the classification of wiping tasks to evaluate our reasoning methods with practical examples. Now, with the theoretical insights of our classification of compliant manipulation tasks, we are able to conclude that all three actions allow to share one control strategy, thanks to the fact that they share the same contact situation as defined in Fig. 2 (External Contact - Friction - Deformation - Neg. Penetration).

\section{CONCLUSION}

Summarized one can say that the desired effects of a manipulation task have significant influence on the task parameterization on all levels of abstraction. Therefore, we believe that this fact should also be reflected in the classification of manipulation tasks, to guide software engineers in the development and parameterization process. We have proposed such a taxonomy for compliant manipulation tasks by investigating different physical contact situations. Rather than defining our taxonomy on geometric features, we defined a more abstract taxonomy that integrates compliant contact behavior implicitly. To this end, we have sub-categorized wiping tasks to derive a set of generic process models applicable to various situations in human environments. This two-step taxonomy is applicable to concretize actions for application in automated planning. To showcase this, we have outlined how the task of sweeping shards can be formulated w. r. t. our classifications.

So far we have only investigated one concrete class of compliant manipulation in detail, namely wiping tasks. As discussed in Sec. III-C, our taxonomy may have possible influence on the selection of appropriate control strategies. To verify that, a more detailed analysis for each branch of the tree has to be conducted. A possible outcome could be a standard controller, respective, a distinct set of standard parameters for each branch of the proposed classification. These general purpose strategies could be utilized for previously unseen problems if no specialized control strategy is known to the robot. Moreover, a distinct set of parameters for each branch of the tree could be exploited to automate the parameterization process for new actions, new objects, or new environments. Eventually, a complete set of actions covering each aspect of compliant manipulation is desirable, yet even an incomplete set is already valuable. For example, developing generic action templates for each wiping task according to Fig. 3 can already be utilized as basis for 
common cleaning tasks, which cover almost half of the tasks in domestic environments [24].

The proposed classification constitutes one step towards a generalized specification of compliant manipulation tasks for automated planning in robotics. Action templates can be utilized by an expert user to implement the corresponding process models. However, action templates do not represent a formal language which is desirable to describe generic process models in a human comprehensible, high level of abstraction. The existing methods do only partially fulfill the requirements to describe compliant manipulation tasks. To fully make use of our classification, a formal method suitable for automated planning [34], and capable of describing compliant behavior [35] will be necessary in the future.

\section{ACKNOWLEDGMENTS}

This work was partially funded by the European Communitys Seventh Framework Programme under grant agreement no. 608849 EuRoC and partially by the Helmholtz Association Project HVF-0029 RACE-LAB.

\section{REFERENCES}

[1] M. Ghallab, D. Nau, and P. Traverso, Automated Planning: theory and practice. Morgan Kaufmann, 2004.

[2] M. R. Cutkosky, "On grasp choice, grasp models, and the design of hands for manufacturing tasks," IEEE Transactions on Robotics and Automation, vol. 5, no. 3, pp. 269-279, 1989.

[3] I. M. Bullock, R. R. Ma, and A. M. Dollar, "A hand-centric classification of human and robot dexterous manipulation," IEEE Transactions on Haptics, vol. 6, no. 2, pp. 129-144, 2013.

[4] J. D. Morrow and P. K. Khosla, "Manipulation task primitives for composing robot skills," in Proc. of the IEEE International Conference on Robotics and Automation (ICRA), vol. 4. IEEE, 1997, pp. 33543359.

[5] S. Stansfield, "Robotic grasping of unknown objects: A knowledgebased approach," The International journal of robotics research, vol. 10, no. 4, pp. 314-326, 1991.

[6] M. Grebenstein, A. Albu-Schäffer, T. Bahls, M. Chalon, O. Eiberger, W. Friedl, R. Gruber, S. Haddadin, U. Hagn, R. Haslinger, et al., "The dlr hand arm system," in Proc. of the IEEE International Conference on Robotics and Automation (ICRA), 2011, pp. 3175-3182.

[7] I. A. Kapandji and L. H. Honoré, The physiology of the joints: annotated diagrams of the mechanics of the human joints. E. \& S. Livingstone London, 1970, vol. 1.

[8] M. Chalon, A. Dietrich, and M. Grebenstein, "The thumb of the anthropomorphic awiwi hand: From concept to evaluation," International Journal of Humanoid Robotics (IJHR), vol. 11, 2014.

[9] T. Feix, R. Pawlik, H.-B. Schmiedmayer, J. Romero, and D. Kragic, "A comprehensive grasp taxonomy," in Robotics, Science and Systems: Workshop on Understanding the Human Hand for Advancing Robotic Manipulation, 2009, pp. 2-3.

[10] A. Bloomfield, Y. Deng, J. Wampler, P. Rondot, D. Harth, M. McManus, and N. Badler, "A taxonomy and comparison of haptic actions for disassembly tasks," in Proc. of the Virtual Reality Conference, 2003, pp. 225-231.

[11] F. Worgotter, E. E. Aksoy, N. Kruger, J. Piater, A. Ude, and M. Tamosiunaite, "A simple ontology of manipulation actions based on hand-object relations," IEEE Transactions on Autonomous Mental Development, vol. 5, no. 2, pp. 117-134, 2013.

[12] M. K. Vukobratović and V. Potkonjak, "Dynamics of contact tasks in robotics. part i: general model of robot interacting with environment," Mechanism and machine theory, vol. 34, no. 6, pp. 923-942, 1999.

[13] J. Liu, F. Feng, Y. Nakamura, and N. S. Pollard, "A taxonomy of everyday grasps in action," in IEEE-RAS International Conference on Humanoid Robots (ICHR), 2014, pp. 573-580.

[14] D. Leidner, C. Borst, and G. Hirzinger, "Things are made for what they are: Solving manipulation tasks by using functional object classes," in Proc. of the IEEE/RAS International Conference on Humanoid Robots (ICHR), 2012, pp. 429-435.
[15] A. Dietrich, T. Wimböck, A. Albu-Schäffer, and G. Hirzinger, "Reactive whole-body control: Dynamic mobile manipulation using a large number of actuated degrees of freedom," IEEE Robotics \& Automation Magazine, vol. 19, no. 2, pp. 20-33, 2012.

[16] D. Leidner, A. Dietrich, F. Schmidt, C. Borst, and A. Albu-Schäffer, "Object-centered hybrid reasoning for whole-body mobile manipulation," in Proc. of the IEEE International Conference on Robotics and Automation (ICRA), 2014, pp. 1828-1835.

[17] D. Williams and O. Khatib, "The virtual linkage: A model for internal forces in multi-grasp manipulation," in Proc. of the IEEE International Conference on Robotics and Automation, 1993, pp. 1025-1030.

[18] H. Urbanek, A. Albu-Schäffer, and P. van der Smagt, "Learning from demonstration: repetitive movements for autonomous service robotics," in Proc. of the IEEE/RSJ International Conference on Intelligent Robots and Systems (IROS), 2004, pp. 3495-3500.

[19] D. Leidner, S. Music, and A. Wedler, "Robotic deployment of extraterrestrial seismic networks," in Proc. of the 13th Symposium on Advanced Space Technologies in Robotics and Automation (ASTRA), 2015.

[20] T. Wimböck, B. Jahn, and G. Hirzinger, "Synergy level impedance control for multifingered hands," in Proc. of the IEEE/RSJ International Conference on Intelligent Robots and Systems (IROS), September 2011, pp. 973-979.

[21] A. Stemmer, A. Albu-Schäffer, and G. Hirzinger, "An analytical method for the planning of robust assembly tasks of complex shaped planar parts," in Proc. of the IEEE International Conference on Robotics and Automation (ICRA), April 2007, pp. 317-323.

[22] N. Hogan, "Stable execution of contact tasks using impedance control," in Proc. of the IEEE International Conference on Robotics and Automation, vol. 4, 1987, pp. 1047-1054.

[23] Y. Xie, D. Sun, C. Liu, H. Tse, and S. Cheng, "A force control approach to a robot-assisted cell microinjection system," International Journal of Robotics Research, vol. 29, no. 9, pp. 1222-1232, 2010.

[24] M. Cakmak and L. Takayama, "Towards a comprehensive chore list for domestic robots," in Proc. of the ACM/IEEE International Conference on Human-Robot Interaction (HRI), 2013, pp. 93-94.

[25] M. Ghallab, A. Howe, D. Christianson, D. McDermott, A. Ram, M. Veloso, D. Weld, and D. Wilkins, "Pddl-the planning domain definition language," AIPS98 planning committee, vol. 78, no. 4, pp. $1-27,1998$.

[26] K. D. Forbus, "Qualitative process theory," Artificial intelligence, vol. 24, no. 1, pp. 85-168, 1984.

[27] L. Mösenlechner and M. Beetz, "Parameterizing actions to have the appropriate effects," in IEEE/RSJ International Conference on Intelligent Robots and Systems (IROS). IEEE, 2011, pp. 4141-4147.

[28] P. J. Hayes, The naive physics manifesto. Institut pour les études sémantiques et cognitives/Université de Genève, 1978.

[29] L. Kunze, M. E. Dolha, E. Guzman, and M. Beetz, "Simulation-based temporal projection of everyday robot object manipulation," in Proc. of the International Conference on Autonomous Agents and Multiagent Systems (AAMAS), 2011, pp. 107-114.

[30] M. Tenorth, U. Klank, D. Pangercic, and M. Beetz, "Web-enabled robots," IEEE Robotics \& Automation Magazine, vol. 18, no. 2, pp. 58-68, 2011.

[31] M. Tenorth, A. Perzylo, R. Lafrenz, and M. Beetz, "The roboearth language: Representing and exchanging knowledge about actions, objects, and environments," in Proc. of the IEEE International Conference on Robotics and Automation (ICRA), 2012, pp. 1284-1289.

[32] D. Leidner and A. Dietrich, "Towards intelligent compliant service robots," in Twenty-Ninth AAAI Conference on Artificial Intelligence, AAAI Video Competition, 2015. [Online]. Available: http://youtu.be/jgIwgcz8iaM

[33] D. Leidner, A. Dietrich, M. Beetz, and A. Albu-Schäffer, "Knowledgeenabled parameterization of whole-body control strategies for forcesensitive tasks," cond. accepted for publication in Autonomous Robots (AURO): Special Issue on Whole-Body Control of Contacts and Dynamics for Humanoid Robots, 2015.

[34] G. Bartels, I. Kresse, and M. Beetz, "Constraint-based movement representation grounded in geometric features," in Proceedings of the IEEE/RAS International Conference on Humanoid Robots (ICHR), 2013, pp. 547-554.

[35] G. Borghesan and J. De Schutter, "Constraint-based specification of hybrid position-impedance-force tasks," in Proc. of the IEEE International Conference on Robotics and Automation (ICRA), 2014, pp. 2290-2296. 